\title{
La regolazione dell'equilibrio acido-base nei pazienti in trattamento emodialitico
}

\author{
D. Cresseri, B. Ravasi, G. Valenti, G. Buccianti \\ Servizio di Nefrologia e Dialisi IRCCS \\ Ospedale Maggiore Policlinico di Milano
}

\section{Fisiopatologia dell'acidosi metabolica uremica}

La fase avanzata dell'uremia è caratterizzata da molteplici alterazioni dell'equilibrio idroelettrolitico ed in particolare da acidosi metabolica di grado progressivo.

La comparsa di tale anomalia determina una serie di effetti a carico di numerosi organi ed apparati (Tab. I): a carico del sistema cardiovascolare la diminuzione del $\mathrm{pH}$ plasmatico induce una riduzione della contrattilità miocardica, disturbi del ritmo, costrizione venosa e riduzione del tono arteriolare; a carico del sistema nervoso centrale (SNC) l'acidosi grave comporta un progressivo obnubilamento del sensorio fino allo stato di coma; mentre, dal punto di vista biochimico, sono state descritte numerose alterazioni quali la riduzione della sensibilità cellulare all'insulina (1), la depressione della glicolisi anaerobia e la ridotta utilizzazione celebrale della glutamina (2).

L'acidosi metabolica dell'uremico diviene clinicamente rilevante per valori di filtrato glomerulare (FG) inferiore a 30 $\mathrm{ml} /$ minuto, mentre per valori di FG progressivamente decrescenti dai livelli normali a $30 \mathrm{ml} / \mathrm{min}$ il $\mathrm{pH}$ plasmatico rimane relativamente costante (3). Con il progredire dell'insufficienza renale cro- nica (IRC) il bilancio idrogenionico presenta una positivizzazione valutabile intorno a $20 \mathrm{mEq} / 24 \mathrm{~h}$, pari al $18-20 \%$ della quota globale degli acidi fissi prodotti dal metabolismo endogeno.

La relativa stabilità del $\mathrm{pH}$ plasmatico nelle fasi intermedie dell'IRC è garantita dall'esistenza di un sistema dotato di una grande disponibilità di tamponi in grado di neutralizzare i radicali acidi ritenuti cronicamente: tale sistema è rappresentato dal tessuto osseo contenente circa $35.000 \mathrm{mEq}$ di tamponi disponibili. Il progressivo consumo di tali tamponi è il principale responsabile della progressiva demineralizzazione ossea favorita dalla ridotta attività dell'1 alfa idrossilasi renale con conseguente ridotta sintesi di $1,25(\mathrm{OH})_{2}$ D3 indotta dalla acidosi metabolica (4).

La patogenesi dell' acidosi metabolica uremica dipende da una insufficiente eliminazione dei radicali acidi o da una ridotta capacità tubulare di riassorbimento dei bicarbonati???

L'osservazione che le urine prodotte dal soggetto con IRC di grado medio elevato sono abitualmente acide indicherebbe una conservata capacità dei nefroni superstiti residui di riassorbire il bicarbonato filtrato. Tuttavia, parallelamente alla distruzione nefronica si riduce l'escrezione urinaria di ioni ammonio nonostante che la presenza di acidosi metabolica rappresenti uno stimolo alla produzione endocellulare. La riduzione dell'e- screzione ammoniacale procede in modo proporzionale alla caduta del filtrato e sembra essere indipendente alla malattia di base, mentre l'escrezione di acidità titolabile appare compromessa in minor misura rispetto all'ammoniogenesi.

\section{Tecniche dialitiche e controllo dell'acidosi metabolica}

L'IRC terminale è caratterizzata da una progressiva e crescente ritenzione di acidi generati dal metabolismo intermedio o assorbiti dall'intestino, come l'acido carbonico, gli acidi metabolizzabili (aceto-acetato e chetoacidi) e gli acidi non metabolizzabili (solforico, cloridrico e fosforico) (Tab. III).

Il trattamento sostitutivo soddisfa l'esigenza di eliminare o tamponare tali valenze acide, sia tramite trasporto diffusivo dal plasma al bagno di dialisi degli acidi non metabolizzabili sia aggiungendo nuove sostanze tampone al plasma.

La scelta del tampone da somministrare al paziente caratterizza le diverse strategie dialitiche ed ha subito nel corso degli anni adattamenti e miglioramenti allo scopo di raggiungere un equilibrio il più vicino possibile al fisiologico (Tab. IV). Il primo tampone proposto per la correzione dell'acidosi uremica risale al 1947, 
TAB. I - EFFETTI DELLA ACIDOSI METABOLICA

\begin{tabular}{|c|c|}
\hline Organi ed apparati & Effetti \\
\hline SISTEMA CARDIOVASCOLARE & $\begin{array}{l}\text { - Riduzione della contrattilità miocardica } \\
\text { - Disturbi del ritmo } \\
\text { - Costrizione venosa } \\
\text { - Riduzione del tono arteriolare }\end{array}$ \\
\hline SISTEMA NERVOSO CENTRALE & - Obnubilamento del sensorio fino al coma \\
\hline SISTEMA RESPIRATORIO & $\begin{array}{l}\text { - Stimolazione del centro respiratorio e della ventilazione } \\
\text { alveolare con diminuzione della } \mathrm{pO}_{2} \\
\text { - Vasocostrizione delle arteriole polmonari } \\
\text { - Alterazioni dell'affinità } \mathrm{Hb} / \mathrm{O}_{2}\end{array}$ \\
\hline$\underline{\text { SISTEMA DIGESTIVO }}$ & - Anoressia e vomito \\
\hline METABOLISMO OSSEO & $\begin{array}{l}\text { - Ridotta sintesi di } 1,25,(\mathrm{OH})_{2} \text { Vit. D3 } \\
\text { - Aumento della increzione di PTH } \\
\text { - Aumento della sensibilità ossea al PTH } \\
\text { - Demineralizzazione ossea }\end{array}$ \\
\hline$\underline{\mathrm{BIOCHIMICA}}$ & $\begin{array}{l}\text { - Riduzione della sensibilità cellulare all'insulina } \\
\text { - Depressione della glicolisi anaerobia } \\
\text { - Ridotta utilizzazione cerebrale di glutamina }\end{array}$ \\
\hline
\end{tabular}

ed era rappresentato dal bicarbonato.

La scelta di tale tampone fisiologico venne tuttavia abbandonata a causa delle molteplici difficoltà tecniche di preparazione rappresentate dalla tendenza del calcio e del magnesio a precipitare a $\mathrm{pH}$ alcalino. Negli anni ' 60 venne proposta e successivamente ampiamente diffusa la sostituzione del tampone bicarbonato con l'acetato, che garantiva anch'esso una soddisfacente correzione dell'acidosi uremica evitando al tempo stesso gli inconvenienti tecnici precedentemente descritti (5).

La grande diffusione e la semplificazione del trattamento dialitico che si ebbe negli anni '70 è probabilmente da attribuire per buona parte all'utilizzo del tampone acetato ed alla facilità di preparazione del bagno di dialisi che ne consegue.

\section{Acetato dialisi}

Nonostante la semplificazione tecnica connessa con l'impiego del tampone acetato, tale tipo di dialisi comporta una serie di problemi biochimico-clinici di peso rilevante (Tab. II).

Per ottenere una correzione ottimale dell'acidosi metabolica uremica la cessione di acetato dal bagno di dialisi al paziente dovrebbe avvenire parallelamente al rimpiazzo dei bicarbonati perduti. In realtà in acetato-dialisi le possibilità di correzione dell'acidosi sono condizionate da diversi fattori:

1) la perdita dei bicarbonati dal plasma al dialisato che ne è privo, 2) la velocità del passaggio di acetato dal dialisato al plasma, 3) la velocità di metabolizzazione dell'acetato.
Nel soggetto normale la capacità di trasformare acetato a bicarbonato raggiunge le $300 \mathrm{mMol} / \mathrm{h}$ (6), mentre negli uremici tale possibilità è ridotta a 200-250 $\mathrm{mMol} / \mathrm{h}(7,8)$. Con gli usuali flussi dialitici si raggiungono e si superano rapidamente le capacità metaboliche massime, ottenendosi elevati valori di acetatemia. Tale sovraccarico è responsabile di alcune complicanze intradialitiche, tra cui l'ipotensione per le proprietà vasodilatatrici del tampone stesso, l'ipossia di non chiara origine e la diversione metabolica dell'acetato.

È stato infatti osservato che l'acetato infuso può sfuggire alla metabolizzazione ad acetil-CoA ed essere deviato verso la sintesi finale di lipidi ed acidi organici, collaborando alla creazione di uno stato di dislipidemia favorente complicanze a livello dell'apparato cardiovascolare.

\section{TAB. II - PROBLEMI BIOCHIMICO-CLINICI DELL'ACETATO-DIALISI}

- Mancata correzione dell'equilibrio acido-base
- Possibile diversione metabolica dell'acetato
- Impossibilità di metabolizzare completamente l'acetato
- Ipossia sintomatica in taluni pazienti

\section{Bicarbonato dialisi}

La progressiva sostituzione del tampone acetato con il bicarbonato ha probabilmente rappresentato la maggiore innovazione tecnica degli anni '80 ed ha avuto una graduale espansione nella maggio- 


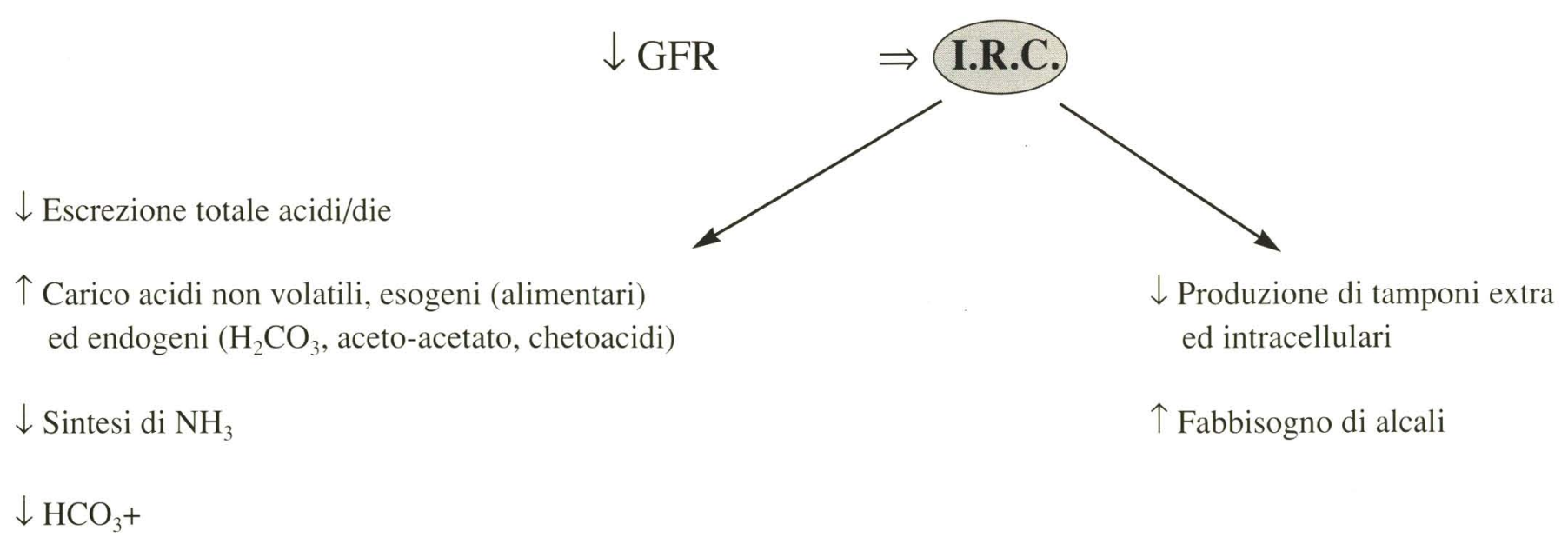

Ritenzione anioni solfati: $\mathrm{SO}_{4}+2 \mathrm{H}^{+} \rightarrow \mathrm{H}_{2} \mathrm{SO}_{4}$

$$
\begin{aligned}
\text { fosfati: } \mathrm{PO}_{4}+2 \mathrm{H}^{+} & \rightarrow \mathrm{H}_{2} \mathrm{PO}_{4} \\
\text { cloridrici: } \mathrm{Cl}^{-}+\mathrm{H}^{+} & \rightarrow \mathrm{HCl}
\end{aligned}
$$$$
\downarrow
$$$$
\text { ACIDOSI METABOLICA }
$$

ranza dei centri dialisi. Il suo impiego ha coinciso con una riduzione significativa di una serie di disturbi clinici intradialitici. L'incidenza degli episodi ipotensivi si è ridotta e sembra essere direttamente correlata con 1'abbandono dell'acetato. Tale tampone infatti, somministrato per via venosa, induce vasodilatazione e depressione della contrattilità miocardica, accanto ad una alterata risposta dei recettori adrenergici. Tutto ciò si aggiunge all'ipotensione indotta dalla sottrazione volemica. L'adozione del bicarbonato elimina talune peculiarità metaboliche proprie dell'acetato e concorre a rendere più confortevole il trattamento dialitico mediante una riduzione anche di altri sintomi quali la nausea, il vomito, i crampi, la cefalea e l'astenia postdialitica. Questi sintomi si correlano direttamente con la concentrazione plasmatica dell' acetato e del suo metabolita isocitrato.

Per quanto riguarda più da vicino gli effetti sull'equilibrio acido-base, il pH ematico e la bicarbonatemia aumentano progressivamente durante la seduta diali- tica. Alla fine della dialisi è spesso evidenziabile una condizione di moderata alcalosi la cui entità dipende dal bilancio totale dei bicarbonati e dallo stato predialitico del $\mathrm{pH}$. Il $\mathrm{pH}$ plasmatico e la bicarbonatemia tendono a ridursi gradualmente nell'intervallo dialitico.

La bicarbonato dialisi, infine, attraverso una migliore correzione dell'acidosi metabolica può avere un effetto benefico sulla mineralizzazione ossea riducendo il consumo di basi prelevate dal tessuto osseo per il tamponamento delle valenze acide.

\section{Emodiafiltrazione e biofiltrazione}

Negli ultimi anni il trattamento depurativo è stato oggetto di numerosi miglioramenti che hanno determinato la comparsa di metodiche sostitutive alternative al trattamento tradizionale.

La prima dimostrazione della possibilità di utilizzare un trattamento depurativo misto di ultrafiltrazione ed emodialisi si deve a Kunitomo nel 1977 (9); ma fu Leber nel 1978 che perfezionò una tecnica in grado di utilizzare contemporaneamente la depurazione diffusiva e convettiva superando i limiti di entrambe (10). Si diffuse quindi progressivamente l'emodiafiltrazione (EDF) caratterizzata dalla sottrazione di elevate quantità di acqua plasmatica grazie all'impiego di membrane ad elevata permeabilità idraulica ed all'infusione di liquidi di sostituzione in misura minore all'emofiltrazione $(\mathrm{HF})$ utilizzando il trasporto diffusivo per la depurazione dei soluti a basso peso molecolare.

La quantità di liquido reinfuso può variare in EDF oscillando dai 3 litri dei trattamenti "soft" ai 18 litri dei trattamenti "hard". Il tampone contenuto nel liquido di sostituzione è generalmente rappresentato dal lattato in concentrazioni di 40-42 $\mathrm{mMol} / 1$, mentre è consigliabile impiegare il tampone bicarbonato nel bagno di dialisi poiché, con l'impiego di membrane ad elevata permeabilità idrau- 
TAB. IV - TRATTAMENTI SOSTITUTIVI EMODIALITICI ED ACIDOSI METABOLICA

TIPI DI EMODIALISI (HD)

HD STANDARD HD BICARBONATO

EMODIAFILTRAZIONE+

BIOFILTRAZIONE

BIOFILTRAZIONE

(AFB)

\begin{tabular}{|c|c|c|c|c|}
\hline $\begin{array}{l}\text { Tampone } \\
\text { bagno HD }\end{array}$ & Acetato & $\begin{array}{l}\text { Bicarbonato }+ \\
4-5 \mathrm{mEq} / \mathrm{L} \text { Acetato }\end{array}$ & $\begin{array}{l}\text { Bicarbonato o } \\
\text { Acetato }\end{array}$ & Nessuno \\
\hline $\begin{array}{l}\text { Tampone } \\
\text { liquido di } \\
\text { infusione }\end{array}$ & // & // & $\begin{array}{l}\text { Bicarbonato o } \\
\text { Lattato }\end{array}$ & Bicarbonato \\
\hline$\underline{\text { Vantaggi }}$ & $\begin{array}{l}\text { Semplicità di preparazione } \\
\text { del bagno di dialisi } \\
\text { Basso costo }\end{array}$ & $\begin{array}{l}\text { Fisiologica correzione } \\
\text { della acidosi } \\
\downarrow \text { Ipotensione intradialitica } \\
\downarrow \text { Episodi di nausea, } \\
\quad \text { vomito, crampi }\end{array}$ & $\begin{array}{l}\text { Buona tolleranza } \\
\text { dialitica } \\
\text { Buona correzione } \\
\text { dell'acidosi }\end{array}$ & $\begin{array}{l}\text { Correzione } \\
\text { personalizzata } \\
\text { dell'acidosi metabolica } \\
\text { Ottima tolleranza } \\
\text { dialitica }\end{array}$ \\
\hline$\underline{\text { Svantaggi }}$ & $\begin{array}{l}\text { Rischio di incompleta } \\
\text { metabolizzazione del } \\
\text { tampone con conseguente } \\
\text { ipossia, ipotensione } \\
\text { intradialitica, diversione } \\
\text { metabolica dell'acetato } \\
\text { (iperlipemia), depressione } \\
\text { contrattilità miocardica }\end{array}$ & $\begin{array}{l}\text { Maggiore complessità } \\
\text { della preparazione del } \\
\text { bagno di dialisi } \\
\downarrow \text { Formazione di } \\
\text { incrostazioni per } \\
\text { precipitazione di sali } \\
\text { insolubili di } \mathrm{Ca}_{2} \mathrm{HOC}_{3} \\
\text { Rischio di alcalosi post-HD }\end{array}$ & $\begin{array}{l}\uparrow \text { Costo/seduta HD } \\
>\text { complessità gestazionale }\end{array}$ & $\uparrow$ Costo/seduta HD \\
\hline
\end{tabular}

lica il mass transfer di tamponi dal bagno di dialisi al compartimento ematico è estremamente elevato. Ciò comporta, utilizzando bagni ad acetato, il rischio di pericolosi innalzamenti della acetatemia per il superamento della soglia di metabolizzazione, con conseguente comparsa di effetti collaterali quali ipossia (11), riduzione delle resistenze periferiche ed ipotesione (12) e depressione della contrattilità miocardica (13).

\section{Biofiltrazione}

La biofiltrazione (BF) venne sperimentata e diffusa sin dall'inizio degli anni ottanta. Essa rappresentava una semplificazione della classica emodiafiltrazione ed era caratterizzata dalla riduzione dei volumi di reinfusione e dall'impiego quale tampone del liquido di reinfusione del bicarbonato.

Dal punto di vista tecnico la BF consiste in una dialisi ad acetato con l'impie- go di dializzatori di poliacrilonitrile ad elevata permeabilità idraulica ed infusione in post-diluizione di una soluzione di bicarbonato di sodio a concentrazione variabile.

La scelta della qualità della soluzione di infusione dipende in larga parte dall'entità della acidosi metabolica del paziente; pertanto nei soggetti francamente acidotici possono rendersi necessarie infusioni di circa tre litri per seduta con soluzioni a concentrazioni di bicarbonato di 100 $\mathrm{mEq} / \mathrm{l}$, mentre nei pazienti con una soddisfacente correzione dello stato acidosico possono essere impiegate con successo soluzioni a minor concentrazione $\mathrm{di}$ bicarbonato $(40-60 \mathrm{mEq} / \mathrm{l})$ eventualmente aumentando i volumi di infusione.

L'adozione di questa nuova metodica dialitica consente di raggiungere alcuni interessanti obbiettivi: può essere ridotta la durata del trattamento a circa 3-3,30 ore (14) e grazie alla somministrazione intradialitica di una considerevole quota di bicarbonato è possibile ottenere un progressivo innalzamento dei valori di
$\mathrm{pH}$ e bicarbonatemia predialitici con correzione delle più gravi forme di acidosi scompensata.

La valutazione clinica dei risultati a lungo termine ottenuti con la biofiltrazione ha permesso di individuare un netto miglioramento della stabilità cardiovascolare con riduzione significativa degli episodi ipotensivi intradialitici (15), una riduzione delle aritmie intradialitiche ed un miglioramento della funzionalità miocardica valutato attraverso il calcolo della velocità media di accorciamento delle fibre circonferenziali del ventricolo sinistro (VCF) che appare significativamente aumentato (15). I pazienti caratterizzati da spiccato ipercatabolismo e marcata acidosi metabolica presentano generalmente un rapido miglioramento delle condizioni generali con miglioramento dell'anoressia, dell'astenia e dei crampi muscolari, e di conseguenza con possibile aumento del peso secco. Tra le complicanze conseguenti alla metodica in questione segnaliamo rari casi di alcalosi metabolica post-dialitica compensata 
dalla assenza di bicarbonato nel bagno che permette la rimozione diffusiva dei bicarbonati qualora la loro concentrazione plasmatica sia eccessiva. È tuttavia consigliabile, soprattutto nelle fasi iniziali del trattamento, uno stretto controllo dei parametri emogasanalitici al fine di adeguare l'infusione dei bicarbonati.

\section{Biofiltrazione senza acetato $(A F B)$}

La biofiltrazione senza acetato (AFB) rappresenta una evoluzione della emodiafiltrazione ed è caratterizzata dalla assenza di tamponi nel bagno di dialisi, mentre il fabbisogno in basi è garantito dalla infusione in post-diluizione di una soluzione a concentrazione nota di bicarbonato di sodio.

La AFB riunisce in sé i vantaggi metabolici della bicarbonato dialisi (prevenzione della ipossiemia intradialitica, buona correzione della acidosi uremica) ad una elevata semplicità tecnica ed esecutiva. L'assenza del tampone bicarbonato nel bagno di dialisi evita la precipitazione dei carbonati insolubili di calcio e magnesio nei circuiti del preparatore e viene inoltre evitata la presenza nel dialisato di quella piccola quota di acetato (4 $\mathrm{mEq} / \mathrm{l}$ ) indispensabile in bicarbonato dialisi per impedire la precipitazione dei bicarbonati.

La correzione dell'equilibrio acido-base è affídata completamente alla concentrazione dei bicarbonati infusi e pertanto esattamente prevedibile e quantificabile. $\mathrm{Ne}$ consegue che è possibile una personalizzazione del trattamento avvalendosi della conoscenza dei parametri emogasanalitici. È stato calcolato che la quota minima necessaria per compensare le perdite di bicarbonati garantendo un bilancio in pareggio è di circa $800 \mathrm{mEq}$ per seduta. L'impiego di quantità comprese tra 900 e $1000 \mathrm{mEq}$ garantisce un bilancio positivo delle basi. L'adozione della AFB permette quindi una correzione a lungo termine della acidosi soddisfacente ed una ottima tolleranza cardiovascolare del trattamento con riduzione degli episodi di ipotensione senza contemporaneo incremento della pressione predialitica. Inoltre l'impiego di dializzatori ad alta efficienza permette una riduzione dei tempi di trattamento mantenendo una ottima efficacia depurativa.

\section{BIBLIOGRAFIA}

1. De Fronzo RA, Becklis AD Glucose intollerance following chronic metabolic acidosis in man. Am J Physiol 1979; 236: 328.

2. Tizianello A, De Ferrari G, Garibotto G, Gurrieri G. Effects of chronic renal insufficiency and metabolic acidosis on glutammine metabolism in man. Clin Sci Mol Med 1978; 55: 391.

3. Goodman AD, Lemann Jr, Relman AS. Production, excretion and net balance of fixed aced in patients with renal acidosis. J Clin Invest 1965; 44: 495.

4. Sauber B, Garabedian M, Fellot C, Mongin P, Balsan S. The effect of induced metabolic acidosis on vitamin D3 metabolism in rachitic chicks. Calcif Tissue Res 1977; 23: 121 .

5. Mion CM, et al. Substitution of sodium acetate for sodium bicarbonate in the both fluid for hemodialysis. Trans ASAIO 1964; 10: 110.

6. Lundquist S. Production and utilization of free acetate in man. Nature 1962; 193: 579.

7. Kveim M, et al. Utilization of exogenous acetate during hemodialysis. Trans ASAIO 1975; 21: 138.

8. Mausell MA, et al. Incidence and significance of rising blood acetate levels during hemodialysis. Clin Nephrol 1979; 12: 22.

9. Kunitomo T, Lowrie EG, Kumazwo S, O'Brian M, Lazzarus JM, Gottlieb MN, Menil JP. Controlled ultrafiltration (UF) with hemodialysis (HD): analysis of couplin between convective and diffusive mass transfer in a new HD-UF system. Trans ASAIO 1977; 23: 234-43.

10. Leber WW, Wizemann V, Gaubeand G, Rawer P, Schutterle G. Simultaneous hemofiltration in hemodialysis: an effective alternative to hemofiltration and conventional hemodialysis in the treatment of uremic patients. Clin Nephrol 1978: 9: 115-21.

11. Vreman MJ, Assomull VL, Kaiser BA, Blascke TF, Weiner MW. Acetate metabolism and acidbase homeostasis during hemodialysis. Kidney Int 1980; 18 (Suppl. 10): S62-74.

12. Wolff J, Pedersen T, Rossen M, Cleemann-Rasmussen K. Effects of acetate and bicarbonate dialysis on cardiac performance, transmural miocardiac perfusion and acid-base balance. Int J Artif Organs 1986; 9: 105-10.

13. Aizawa $\mathrm{Y}$, Ohmori T, Imai K, Nara Y, Matsuoka M, Mirazawa Y. Depressant action of acetate upon cardiovascular sistem. Clin Nephrol 1977: 8: 477-80.

14. Zucchelli T, Santoro A, Raggiotto G, Degli Esposti E, Sturania A, Capecchi V. Biofiltration in Uremia: Preliminary observations. Blood Purification 1984; 2: 187-95.

15. Bordoni E, Carletti P, Fanculli E, Bibiano L, Lombardo V, Mioli V. Tecnica e clinica della biofiltrazione in: Tecniche nefrologiche e dialitiche 1987, Wichtig Editore 97-105. 